\title{
On time fractional Cahn-Allen equation
}

\author{
Runqing Cui and Yue $\mathrm{Hu}$
}

School of Mathematics and Informatics, Henan Polytechnic University, Jiaozuo 454003, China

Igdcuirunqing@yeah.net, huu3y6@163.com

\section{ABSTRACT}

In [1], Ozkan Güner et al. obtained some exact solutions of the time fractional Cahn-Allen equation.

By using the method proposed in [10], we have tested these solutions and have found that they are not the solutions of this equation.

Keywords: time fractional Cahn-Allen equation; exact solution,Exp-function method; First integral method

\section{SUBJECT CLASSIFICATION 35J05, 35J10, 33E12, 33R11, 35A22 INTRODUCTION}

In [1], Ozkan Güner et al. studied the following time fractional Cahn-Allen equation:

$$
D_{t}^{\alpha} u-u_{x x}-u+u^{3}=0
$$

where $0<\alpha \leq 1$, and

$$
D_{t}^{\alpha} u(x, t)=\frac{1}{\Gamma(1-\alpha)} \frac{d}{d t} \int_{0}^{t}(t-\tau)^{-\alpha}[u(x, \tau)-u(x, 0)] d \tau
$$

Where $D_{t}^{\alpha}$ denotes Jumarie's modified Riemann-Liouville fractional derivative [2]. Eq. (1) arises in many scientific applications such as quantum mechanics and plasma physics [3-8]. They obtained some analytical exact solutions by using Exp-function method, the $\left(G^{\prime} / G\right)$-expansion method and First integral method[3]. However, we have observed that these solutions are not true. Here we list two exact solution obtained in [1] as follows:

$$
\begin{aligned}
& u(x, t)=\frac{1}{2}+\frac{1}{2} \tanh \left(\frac{\sqrt{2}}{2} x+\frac{3}{2 \Gamma(1+\alpha)} t^{\alpha}\right), \\
& u(x, t)=\frac{1}{2}-\frac{1}{2} \tanh \left(\frac{\sqrt{2}}{2} x-\frac{3}{2 \Gamma(1+\alpha)} t^{\alpha}\right) .
\end{aligned}
$$

In section 2, by the method proposed in [10], we will prove that the functions (3) and (4) are not the solutions of the Eq. (1).

\section{ANALYSIS AND RESULTS}

By Eq. (2), we can rewrite the Eq. (1) as: 


$$
\frac{1}{\Gamma(1-\alpha)} \frac{d}{d t} \int_{0}^{t}(t-\tau)^{-\alpha}(u(x, \tau)-u(x, 0)) d \tau=u_{x x}+u-u^{3}
$$

For simplicity, we choose $\alpha=0.5$ in Eq.(1) for checking the obtained solutions (3) and (4).

If the function (4) is a solution of the fractional differential Eq. (1), then the function

$$
u(x, t)=\frac{1}{2}-\frac{1}{2} \tanh \left(\frac{\sqrt{2}}{2} x-\frac{3}{2 \Gamma(1.5)} t^{0.5}\right)
$$

satisfies the following equation:

$$
\frac{1}{\Gamma(0.5)} \frac{d}{d t} \int_{0}^{t}(t-\tau)^{-0.5}(u(x, \tau)-u(x, 0)) d \tau=u_{x x}+u-u^{3}
$$

Integrating both sides of the eq. (7) with respect to $t$ from 0 to 1 , we have

$$
\int_{0}^{1}(1-\tau)^{-0.5}(u(x, \tau)-u(x, 0)) d \tau=\Gamma(0.5) \int_{0}^{1}\left(u_{x x}+u-u^{3}\right) d t
$$

Take $x=0$ in Eq.(8), we have:

$$
\begin{array}{r}
\int_{0}^{1} \frac{1}{2}(1-\tau)^{-0.5} \tanh \left(\frac{3 \tau^{0.5}}{2 \Gamma(1.5)}\right) d \tau \\
=\left.\Gamma(0.5) \int_{0}^{1}\left(u_{x x}+u-u^{3}\right)\right|_{x=0} d t .
\end{array}
$$

By Maple software, we obtain that left side of Eq.(9) approximately equals 0.832725 and right approximately equals 0.087684

Thus, the function (4) is not a solution of the Eq.(1). Similarly we can prove that the function (3) does not satisfy Eq. (1).

\section{DISCUSSION AND CONCLUSIONS}

Different from integer-order differential equation, for a given fractional differential equation, it is very difficulty to test whether or not a function satisfies it. In this paper, by using the method proposed in [11], we have tested the functions (3) and (4) and have found that they are not the solutions of the Eq. (1).

\section{REFERENCES}

[1] Güner O, Bekir A, Cevikel A C. A variety of exact solutions for the time fractional Cahn-Allen equation[J]. The European Physical Journal Plus, 2015, 130(7):1-13.

[2] Jumarie, G., Modified Riemann-Liouville derivative and fractional Taylor series of nondifferentiable functions further 
results, Comput. Math. Appl. 51(2006), pp.1367-1376.

[3] Bekir A, Guner O, Unsal O. The First Integral Method for Exact Solutions of Nonlinear Fractional Differential Equations[J]. Journal of Computational \& Nonlinear Dynamics, 2015, 10(2).

[4] Rawashdeh M S. A reliable method for the space-time fractional Burgers and time-fractional Cahn-Allen equations via the FRDTM[J]. Advances in Difference Equations, 2017, 2017(1):99.

[5] Tariq H, Akram G. New approach for exact solutions of time fractional Cahn-Allen equation and time fractional Phi-4 equation[J]. Physica A Statistical Mechanics \& Its Applications, 2017, 473:352-362.

[6] Esen A, Yagmurlu N M, Tasbozan O. Approximate Analytical Solution to Time-Fractional Damped Burger and Cahn-Allen Equations[J]. Applied Mathematics \& Information Sciences, 2013, 7(5):1951-1956.

[7] Hosseini K, Bekir A, Ansari R. New exact solutions of the conformable time-fractional Cahn-Allen and Cahn-Hilliard equations using the modified Kudryashov method[J]. Optik - International Journal for Light and Electron Optics, 2017, 132:203-209.

[8] Nec Y, Nepomnyashchy A A, Golovin A A. Front-type solutions of fractional Allen-Cahn equation[J]. Physica D Nonlinear Phenomena, 2008, 237(24):3237-3251.

[9] Hou T, Tang T, Yang J. Numerical Analysis of Fully Discretized Crank-Nicolson Scheme for Fractional-in-Space Allen-Cahn Equations[J]. Journal of Scientific Computing, 2017:1-18.

[10]Wang J, Hu Y. On chain rule in fractional calculus[J]. Thermal Science, 2016, 20(3): 803-806.

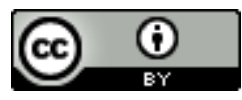

This work is licensed under a Creative Commons Attribution 4.0 International License. 